\title{
Comprehensive study of 28 individuals with SIN3A-related disorder underscoring the associated mild cognitive and distinctive facial phenotype
}

\author{
Meena Balasubramanian ${ }^{1,2} \cdot$ Alexander J. M. Dingemans $^{3}$. Shadi Albaba ${ }^{4} \cdot$ Ruth Richardson $^{5} \cdot$ Thabo M. Yates $^{1}{ }^{1}$. \\ Helen Cox $^{6}$ - Sofia Douzgou ${ }^{7,8} \cdot$ Ruth Armstrong ${ }^{9} \cdot$ Francis H. Sansbury $\mathbb{1}^{10} \cdot$ Katherine B. Burke $^{10}$ • \\ Andrew E. Fry $\mathbb{1 0}^{10}$ - Nicola Ragge ${ }^{6,11}$ - Saba Sharif ${ }^{6}$ - Alison Foster ${ }^{6}$ - Annachiara De Sandre-Giovannoli ${ }^{12,13,14}$. \\ Sahar Elouej ${ }^{12} \cdot$ Pradeep Vasudevan $^{15} \cdot$ Sahar Mansour $^{16} \cdot$ Kate Wilson $^{17} \cdot$ Helen Stewart $^{17} \cdot$ Solveig Heide $^{18}$. \\ Caroline Nava $^{18} \cdot$ Boris Keren $^{18} \cdot$ Serwet Demirdas ${ }^{19} \cdot$ Alice S. Brooks ${ }^{19} \cdot$ Marie Vincent $\mathbb{C}^{20,21} \cdot$ Bertrand Isidor $^{20,21}$. \\ Sebastien Küry $\mathbb{1}^{20,21} \cdot$ Meyke Schouten ${ }^{3}$ - Erika Leenders ${ }^{3}$ - Wendy K. Chung ${ }^{22}$ - Arie van Haeringen ${ }^{23}$. \\ Thomas Scheffner $^{24}$ • Francois-Guillaume Debray ${ }^{25}$. Susan M. White $\mathbb{C}^{26,27} \cdot$ Maria Irene Valenzuela Palafoll $\mathbb{1}^{28}$. \\ Rolph Pfundt ${ }^{3} \cdot$ Ruth Newbury-Ecob ${ }^{29} \cdot$ Tjitske Kleefstra $^{3}$
}

Received: 28 May 2020 / Revised: 15 October 2020 / Accepted: 21 October 2020 / Published online: 12 January 2021

(c) The Author(s) 2021. This article is published with open access

\begin{abstract}
Witteveen-Kolk syndrome (OMIM 613406) is a recently defined neurodevelopmental syndrome caused by heterozygous loss-of-function variants in SIN3A. We define the clinical and neurodevelopmental phenotypes related to SIN3Ahaploinsufficiency in 28 unreported patients. Patients with SIN3A variants adversely affecting protein function have mild intellectual disability, growth and feeding difficulties. Involvement of a multidisciplinary team including a geneticist, paediatrician and neurologist should be considered in managing these patients. Patients described here were identified through a combination of clinical evaluation and gene matching strategies (GeneMatcher and Decipher). All patients consented to participate in this study. Mean age of this cohort was 8.2 years (17 males, 11 females). Out of 16 patients $\geq 8$ years old assessed, eight (50\%) had mild intellectual disability (ID), four had moderate ID (22\%), and one had severe ID $(6 \%)$. Four $(25 \%)$ did not have any cognitive impairment. Other neurological symptoms such as seizures (4/28) and hypotonia (12/28) were common. Behaviour problems were reported in a minority. In patients $\geq 2$ years, three were diagnosed with Autism Spectrum Disorder (ASD) and four with Attention Deficit Hyperactivity Disorder (ADHD). We report 27 novel variants and one previously reported variant. 24 were truncating variants; three were missense variants and one large in-frame gain including exons 10-12.
\end{abstract}

These authors contributed equally: Meena Balasubramanian, Alexander J. M. Dingemans, Shadi Albaba

These authors jointly supervised this work: Meena Balasubramanian, Tjitske Kleefstra

Supplementary information The online version of this article (https:// doi.org/10.1038/s41431-020-00769-7) contains supplementary material, which is available to authorised users.

Meena Balasubramanian

meena.balasubramanian@nhs.net

Extended author information available on the last page of the article

\section{Introduction}

Witteveen-Kolk syndrome (OMIM \#613406) was first described in 2016 with characteristic distinctive facial features, microcephaly, short stature, mild intellectual disability (ID) with delayed cognitive and motor development and subtle anomalies on MRI-brain imaging [1]. Although sparsely reported, frameshift as well as missense variants in the Switch-insensitive 3 transcription regulator family member A (SIN3A) (OMIM *607776) have been described in larger neurodevelopmental disorder cohorts, with an overall mild clinical picture [2]. Narumi-Kishimoto et al. presented a further patient with SIN3A frameshift variant and facial features of Witteveen-Kolk with relatively mild ID and normal growth [3]. 
The SIN3A gene is located in the chromosome 15 band q24 and is within the shortest region of overlap of various reported $15 \mathrm{q} 24$ microdeletions, therefore, is thought to be the critical gene for the atypical $15 q 24$ microdeletion syndrome [4]. SIN3A encodes a transcriptional regulatory protein, which is associated with scaffolding in the core histone deacetylase complex [5]. In our earlier study, we showed that SIN3A is involved in cortical neurogenesis, supporting the hypothesis that variants in the gene that adversely affect its function lead to a broad range of developmental and neurological problems. We identified an additional 28 patients with SIN3A variants in order to comprehensively define the phenotype with a focus on both developmental and behavioural aspects, as well as investigating genotype-phenotype correlations.

\section{Methods}

We collected the molecular and clinical features on 28 unpublished individuals with $\operatorname{SIN} 3 A$ variants by a collaboration facilitated by Deciphering Developmental Disorders (DDD study) [6], GeneMatcher [7] and DECIPHER (DECIPHER v9.24: https://decipher.sanger.ac.uk/) [8], in which, several clinical groups independently identified individuals with developmental delay/intellectual disability (DD/ID) and related phenotypes with rare variants in SIN3A during routine diagnostic genetic testing. An application to the DDD study for a Complementary Analysis Project was made, allowing access to anonymised details of patients with SIN3A variants identified through this study (https://www.ddduk.org/). Clinicians of selected patients were then contacted to invite patients and their families to be recruited. Clinical analysis of these patients was performed during regular consultations focusing on medical history, physical examination and observational analysis of behavioural features along with reported history by the family. In all patients, exome sequencing and variant filtering were performed, according to the routine protocol and diagnostic procedures at each institute.

Identified patients with a class 4 or 5 variants (likely pathogenic or pathogenic variants) in SIN $3 A$, according to the American College of Medical Genetics (ACMG) criteria, were approached to participate in this study. Informed consent for publication was obtained from all patients and/ or their guardians. The responsible clinician reviewed medical records of each participant in order to comprehensively document the phenotype.

The clinical significance of the variants identified was interpreted according to the ACMG [9] guidelines and further review publications [10]. Excluded from the study were: patients with an additional proven genetic diagnosis where the $S I N 3 A$ variant was not thought to be contributory or the sole pathogenic finding, those with a chromosomal anomaly explaining or likely to be explaining the phenotype, and those in whom SIN3A variants were of uncertain clinical significance with no convincing clinical correlation. All the variants reported here have been submitted to DECIPHER database [https://decipher.sanger.ac.uk] and phenotypes are included in DECIPHER database for the DDD patients [Patients 1-13]- DDD identifiers, [https:// decipher.sanger.ac.uk/gene/SIN3A/overview/clinical-info].

\section{Results}

\section{Molecular genetics}

In this study, we reported 28 patients with variants that adversely affect protein function and which are classified as pathogenic or likely pathogenic variants in SIN3A. The findings of this study expand the variant spectrum previously reported in $\operatorname{SIN} 3 A^{, 3}$. 27/28 patients in this study have novel variants. Patient 15 with c.3310C $>$ T, p. (Arg1104*) variant is a family member of a patient we have previously reported in Witteveen et al. [1]. Predominantly, variants found in our patient cohort are truncating and predicted to result in a protein loss-of-function (25/28). All 24 truncating variants have been classified as variants that adversely affect function (pathogenic) using the ACMG and ACGS (The Association for Clinical Genomics Science) variant classification guidelines using criterions: PVS1, PM2, and PS2 where appropriate [9, 10]. Of the four remaining variants, three were missense (patients 4, 18 and 26; Table 1) and one was a large in-frame gain which included the whole of exons 10, 11 and 12 (patient 24; Table 1). These four variants were all de novo in our patients with a specific and consistent phenotype to $\operatorname{SIN} 3 \mathrm{~A}$ and were absent from control population data sets in gnomAD (https://gnomad.broadinstitute.org/). We have also used criterion PM1 at moderate level for two of the three reported missense variants, c.377C $>$ T, p.(Ala126Val) and c.463A $>$ G, p.(Lys155Glu), as they were located within a protein functional domain. These results show that missense causative variants are not clustered in a hot spot within SIN3A (Fig. 1 provides a schematic SIN3A structure and variant locations which, as demonstrated, are distributed throughout the gene). Details of the variants, ACMG criterions and classification are listed in Table 1.

\section{Assessment of pathogenicity of missense variants in SIN3A}

In order to assess if missense variants in our cohort were predicted to adversely affect the critical functional domains in SIN3A, we used a 3D model based on the solution 


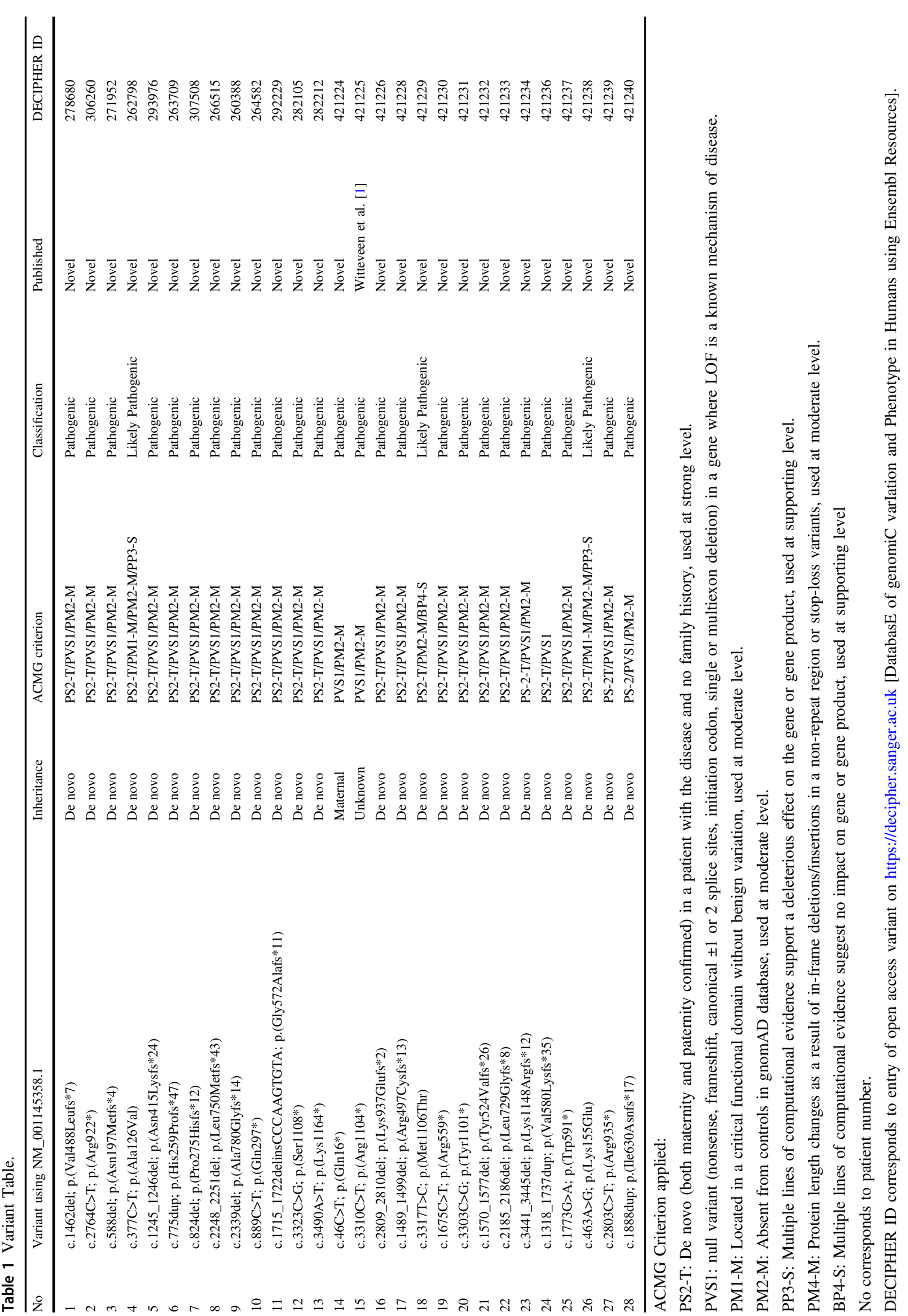



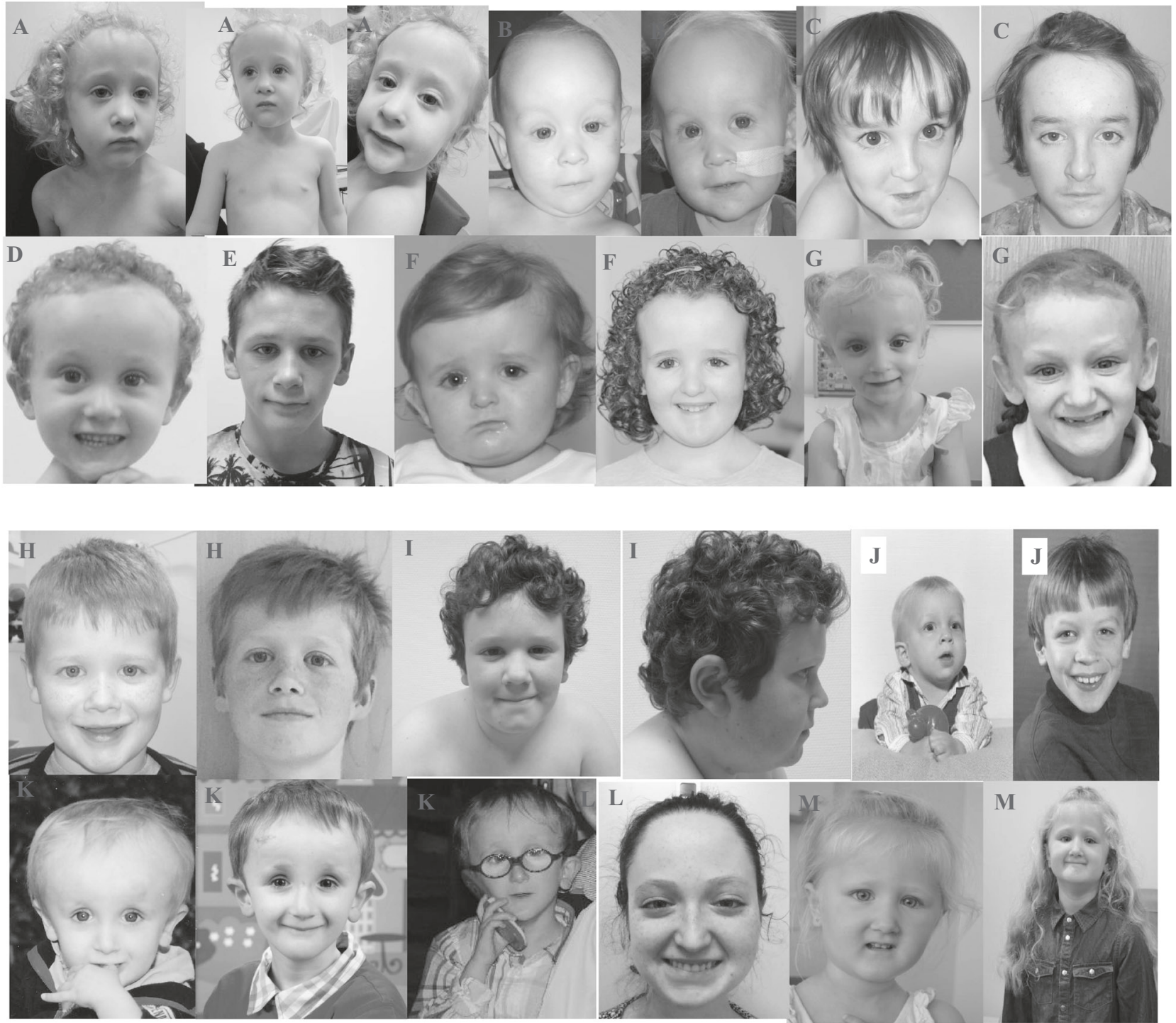

Fig. 1 Characteristic facial appearance of patients with variants in SIN3A. Note the high forehead, small, pointed chin and down-slanting palpebral fissures. A: Patient 1, B Patient 2, C Patient 4, D Patient 5, E
Patient 9, F Patient 11, G Patient 12, H Patient 13, I Patient 16 J Patient 18, K Patient 25, L Patient 27, M Patient 28.

Along with the lack of normal variation in this region (using missense constraint data in SIN3A from Decipher: https:// decipher.sanger.ac.uk/gene/SIN3A\#overview/protein-info), we applied criterion PM1 at moderate level for the classification of both c.377C $>$ T, p.(Ala126Val) and c.463A $>$ G, p.(Lys155Glu). We also suggest that PM1 can be applied at a moderate level for missense variants within residues p.119-189 of the SIN3A protein providing that the change caused by the variant was not present in other species and also meets the PM2 criteria (see Fig. 2).

Finally, the c.3317T>C, p.(Met1106Thr) is within the Cterminal domain of SIN3A, this region is not well characterised and the in silico pathogenicity prediction programs suggests a benign effect (REVEL score of 0.105 shows a benign effect). However, this variant was confirmed to be 


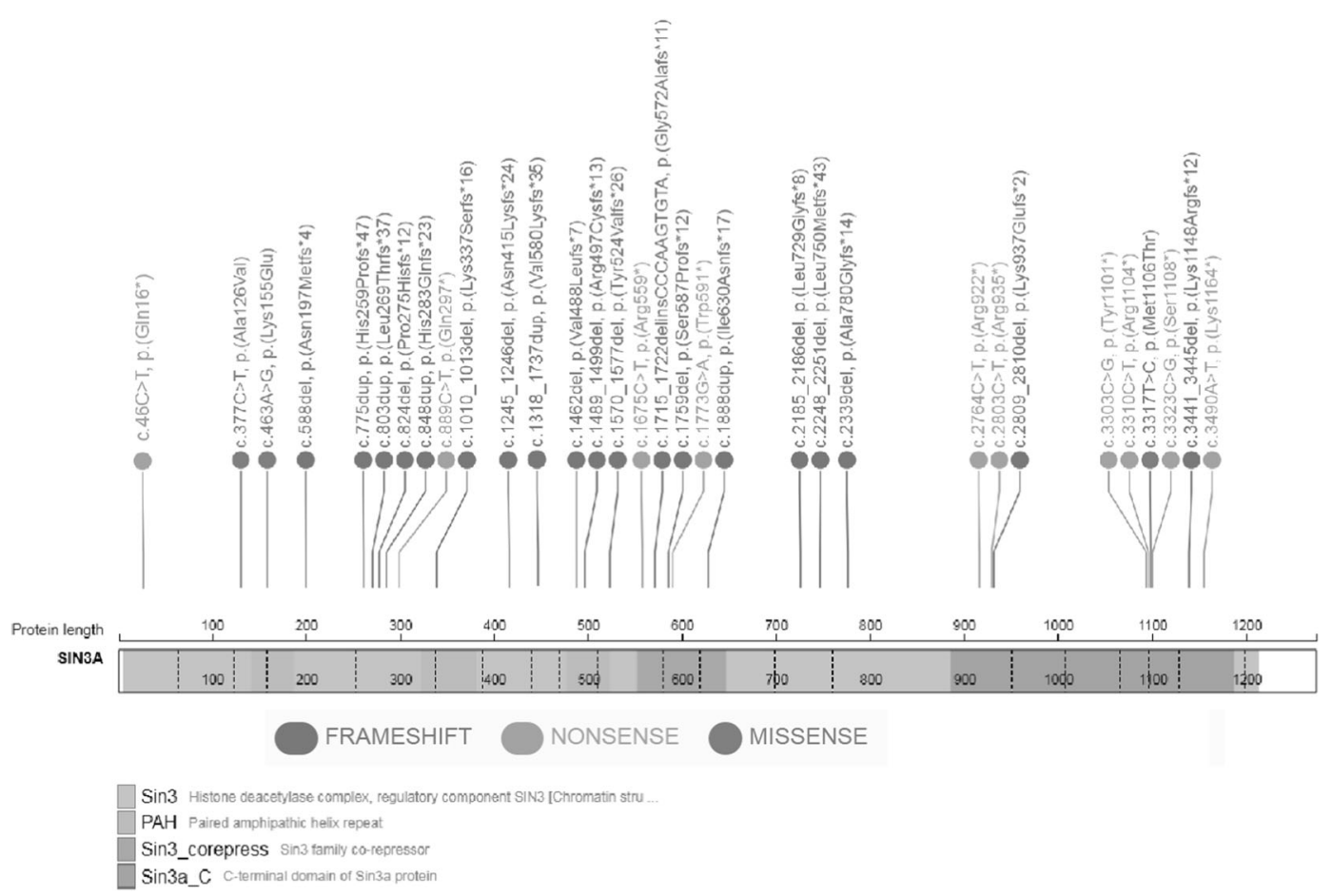

Fig. 2 Schematic SIN3A protein structure and variant location. ITD: intragenic deletion; Plot of variants done using St. Jude Cloud protein paint (https://pecan.stjude.cloud/proteinpaint).

de novo in patient 18, novel in control populations in gnomAD and with a consistent phenotype to that of the rest of our cohort. No other variants were found in this patient on exome sequencing. Therefore, we classified this variant in addition as likely pathogenic, but without the use of PM1.

\section{Clinical findings}

Table 2 shows a summary of the characteristics of 28 patients (17 males, 11 females) with variants in SIN3A who were included in this retrospective study. The mean age of participants was 8.2 years (range 0.6-67 years). Table 3 provides an overview of salient features described in this cohort in comparison to published literature and Table 4 has detailed phenotypic information on this cohort of patients.

\section{Development}

Of 28 patients, 15 (56\%) had global developmental delay. Out of 16 patients $\geq 8$ years old, (one patient was not formally assessed) seven (44\%) had mild intellectual disability (ID), four had moderate ID (25\%), and one had severe ID $(6 \%)$. Four $(25 \%)$ did not appear to have any cognitive impairment. In 21 patients $\geq 5$ years old, some form of motor developmental delay was reported in 13 patients $(62 \%)$ and in 16 patients $(76 \%)$ there was some form of language developmental delay. Intelligence Quotient (IQ) was only formally measured in 6/28 patients and the score ranged from 60 to 100. This suggests that patients within this cohort have low normal intelligence.

Autism spectrum disorder (ASD) is rarely diagnosed before the age of 24 months [14]. In one patient aged 14 months, ASD was thought possible but not formally assessed due to the young age. In three of 23 patients $\geq 2$ years (13\%), ASD was diagnosed. Attention Deficit Hyperactivity Disorder (ADHD) is most commonly diagnosed in children between 6 and 12 years, though this can be diagnosed in a younger age group. ADHD was diagnosed in four patients $\geq 2$ years $(17 \%)$.

\section{Growth and feeding difficulties}

$13 / 28(46 \%)$ patients in our cohort had head circumference at least two standard deviations (SD) below the mean. Weight was less than two SD below the mean in $8 / 28$ patients (29\%) and height was less than two SD below the mean in $5 / 28$ patients (18\%).15/28 (54\%) of patients had feeding difficulties with at least two patients documented to require nasogastric tube feeding mainly in the neonatal period.

\section{Craniofacial features}

Fourteen $(50 \%)$ patients were reported to have craniofacial dysmorphism. In ten patients, the facial gestalt was 


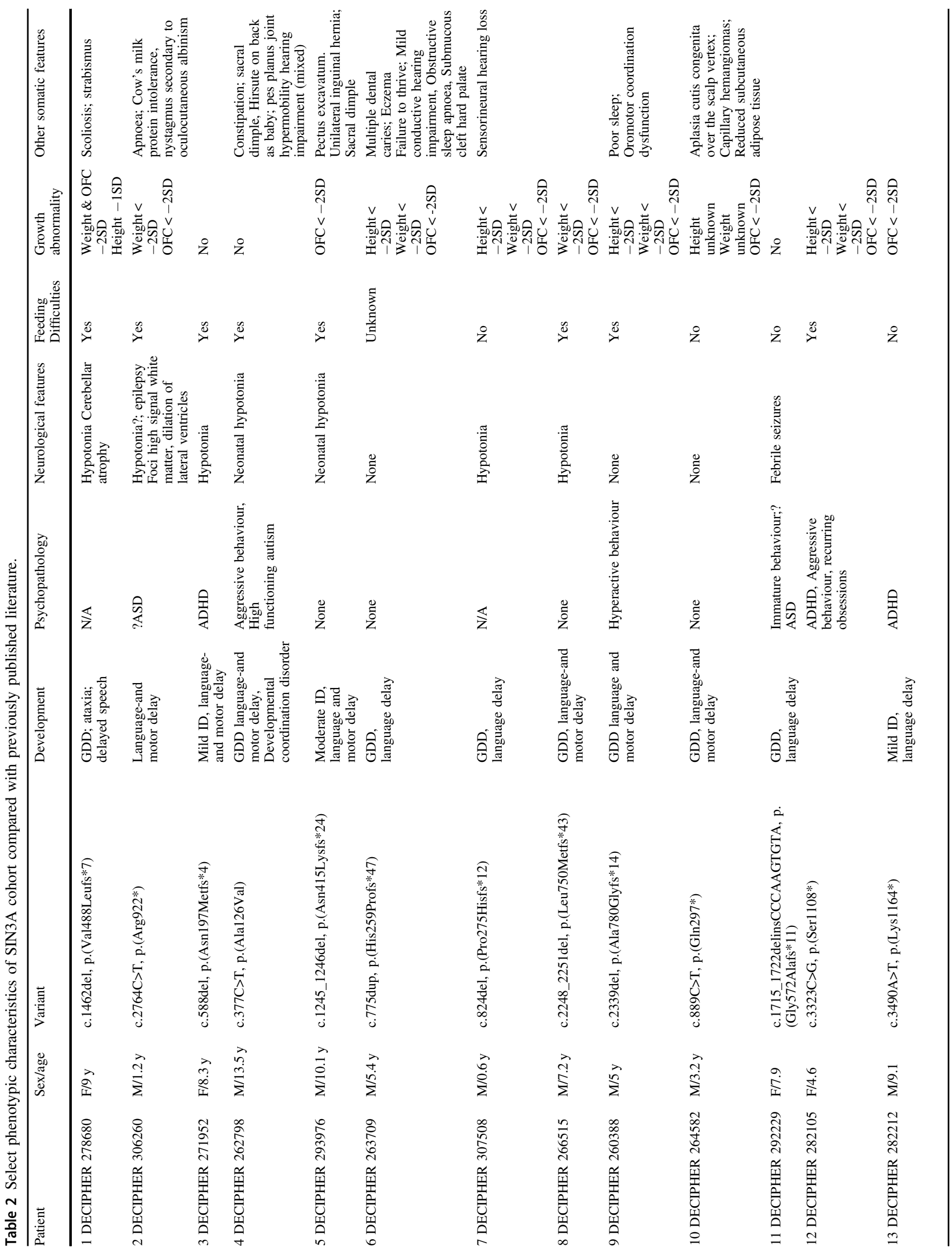




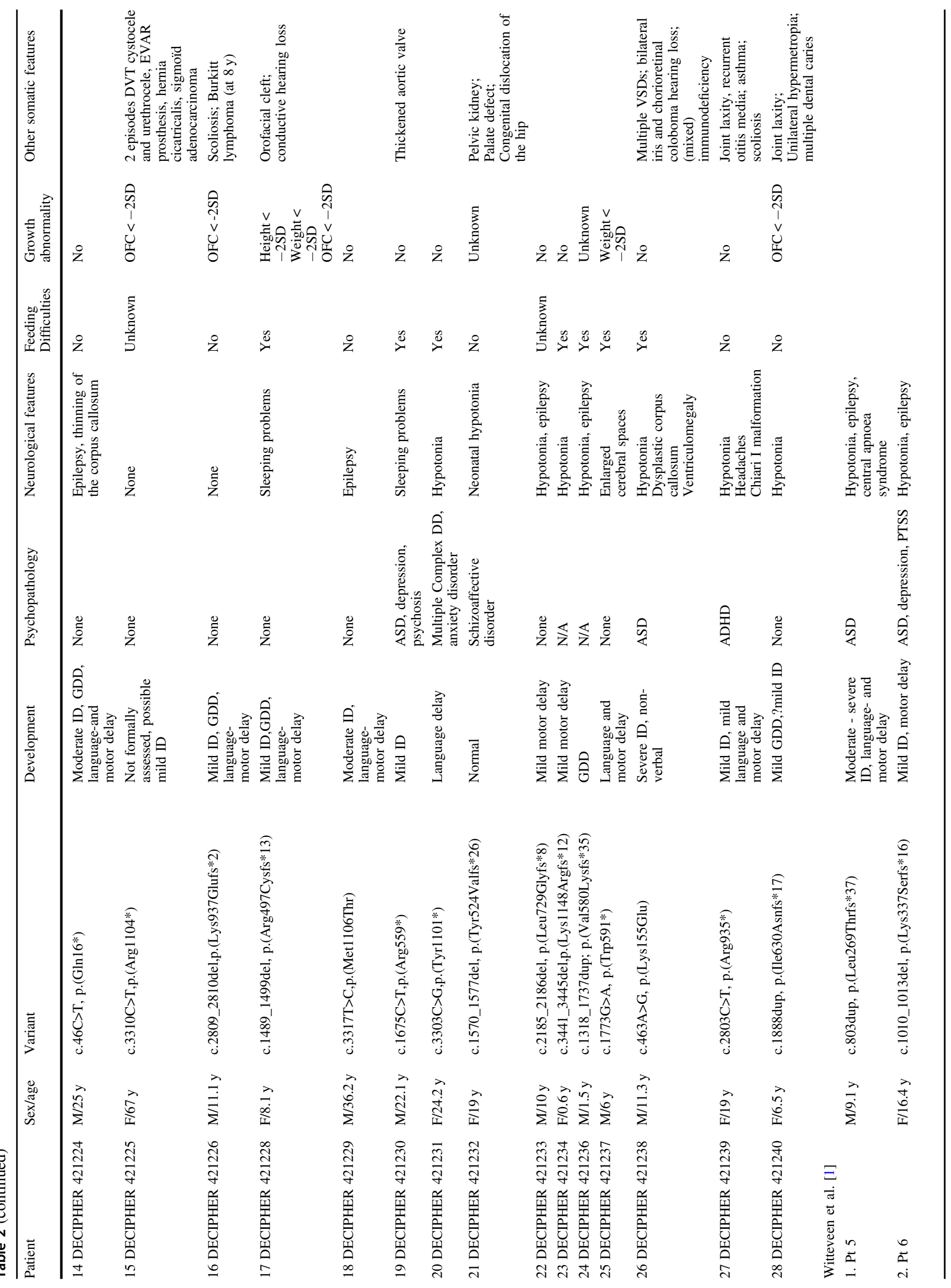




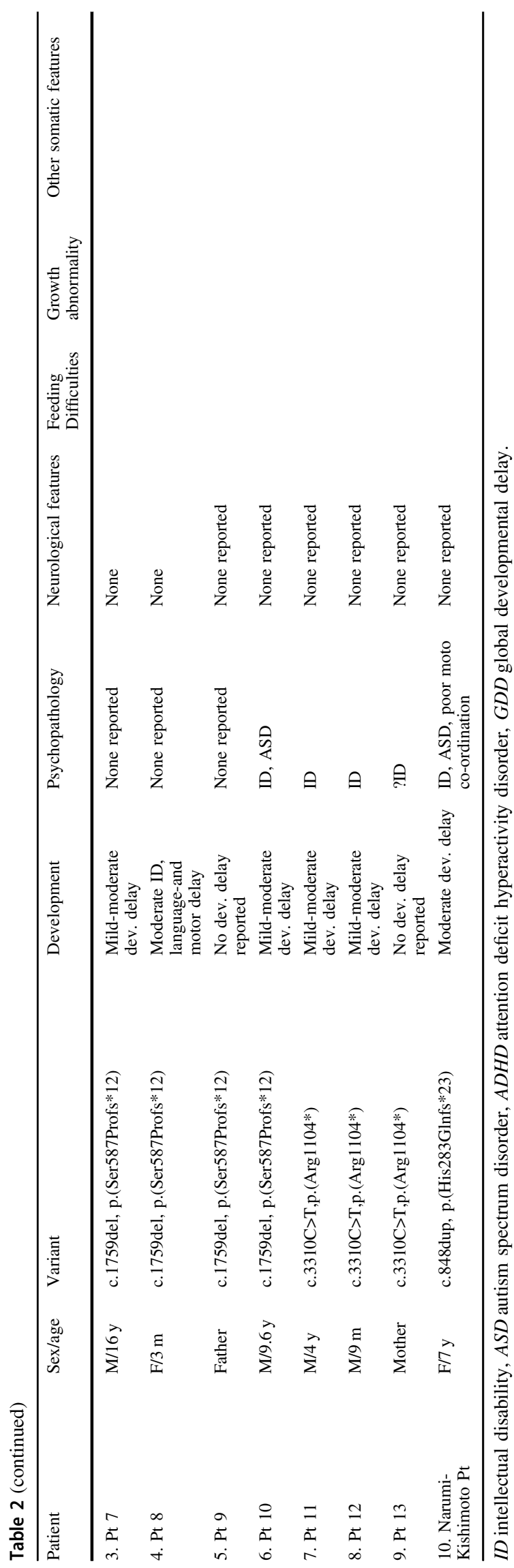

Table 3 Summary of salient features in SIN3A-related disorder based on current cohort and previously published literature.

\begin{tabular}{|c|c|c|c|c|}
\hline Clinical feature & $\begin{array}{l}\text { Current } \\
\text { cohort } \\
(n=28)\end{array}$ & $\begin{array}{l}\text { Witteveen } \\
\text { et al. [1] } \\
(n=9)\end{array}$ & $\begin{array}{l}\text { Narumi- } \\
\text { Kishimoto [3] } \\
(n=1)\end{array}$ & $\begin{array}{l}\text { Total } \\
(n=38)\end{array}$ \\
\hline $\begin{array}{l}\text { Intellectual } \\
\text { disability }\end{array}$ & 16 & 7 & 1 & 24 \\
\hline Mild & 8 & 5 & - & 13 \\
\hline Moderate & 7 & 2 & 1 & 10 \\
\hline Severe & 1 & - & - & 1 \\
\hline Speech delay & 11 & 2 & 1 & 13 \\
\hline Hypotonia & 12 & 2 & - & 14 \\
\hline $\begin{array}{l}\text { Feeding } \\
\text { difficulties }\end{array}$ & 15 & $N / R$ & - & 15 \\
\hline Short stature & 6 & 2 & - & 8 \\
\hline Epilepsy & 4 & 1 & - & 5 \\
\hline $\begin{array}{l}\text { Behavioural } \\
\text { problems }\end{array}$ & 12 & 4 & 1 & 17 \\
\hline
\end{tabular}

confirmed independently by two clinical geneticists with expertise in dysmorphology. Common facial features included a broad, tall forehead; small mouth, thin upper lip with pointed chin and down-slanting palpebral fissures (see Fig. 3). The facial gestalt appears to be similar and potentially recognisable but only in the context of reverse phenotyping with published images, confirming previous findings. Three patients had a palatal defect with one also having a bifid uvula.

\section{Other clinical manifestations}

In terms of neuroimaging, given the milder developmental delay phenotype, many patients in the cohort have not had MRI-brain imaging (19/28, 68\%). Nine out of a total of nine patients that had brain imaging performed had reports available and in 7/9 (78\%) abnormalities were seen. Most common was ventriculomegaly in $2 / 9$, and a hypoplastic/ dysplastic corpus callosum in 2/9. Cerebellar atrophy was reported in two patients and there was one patient had a Chiari 1 malformation.

$6 / 28$ (21\%) patients were noted to have seizures currently or in their past medical history. Hypotonia was a common manifestation as well, present in 12/28 (43\%) patients.

Other reported features included hearing loss (5/28; $18 \%$ ) of which 1 patient had sensorineural hearing loss, two patients had conductive hearing loss and two patients had mixed hearing loss. Ocular abnormalities reported included strabismus (1), nystagmus secondary to ocular albinism (1), bilateral iris and chorioretinal coloboma (1) and hypermetropia (1).

Of note, $2 / 28$ patients were reported to have a malignancy, including sigmoid adenocarcinoma in the 67-year old patient which may be an age-related cancer and 1 younger patient with Burkitt lymphoma (Patient 16 at age 


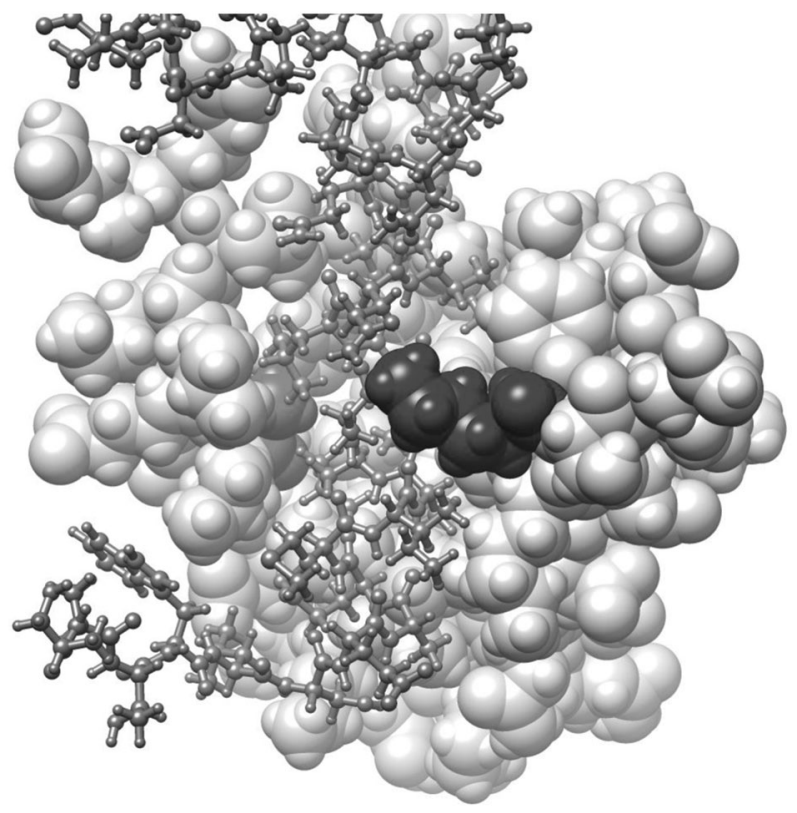

Fig. 3 3D model to demonstrate predicted consequences of SIN3A missense variant in our cohort. This $3 \mathrm{D}$-model is based on the solution structure of mouse Sin3A PAH1 bound to the Sin 3 interaction domain (SID) of SAP25 (Sin3A Associated Protein 25) displayed using UCSF Chimera v1.14 (Pattersen et al. 2004). The PAH1 domain of Sin3A (residues 119-189; sphere model, light grey) with residues Ala126 (red) and Lys155 (blue) highlighted. The SAP25 protein SID domain (residues 126-186; ball and stick model, dark grey) binds in the fold formed by the four helices of Sin3A PAH1. Sin3A Lys155 is predicted to form a $2.2 \mathrm{~A}$ hydrogen bond with the polar side chain of Gln143 of SAP25 (orange line).

eight). Patient 26 was noted to have T-cell lymphopenia at age 11 associated with immunoglobulin deficiency and significant bronchiectasis.

\section{Discussion}

Heterozygous loss-of-function variants in SIN3A were recently described to result in a novel neurodevelopmental syndrome comprising intellectual disability and varying degrees of developmental delay. This syndrome defined as Witteveen-Kolk syndrome was further characterised by subtle brain abnormalities, including corpus callosum dysgenesis and ventriculomegaly, distinctive facial features (a broad, tall forehead; small mouth, thin upper lip with pointed chin and down-slanting palpebral fissures), hyperlaxity and short stature. Furthermore, we showed previously that in vivo functional knockdown of SIN3A leads to reduced cortical neurogenesis, altered neuronal identity and aberrant cortico-cortical projections in the developing mouse brain. Therefore, it is likely that the aberrant cortical development underlies impaired neurodevelopment and leads to cognitive and behavioural problems in patients, varying from paediatric to adult-onset. Here, we summarise the neurodevelopmental and facial phenotype in an additional 28 patients with SIN3A-related disorder.

Table 2 provides an overview of the patients reported here and all previously reported patients with SIN3A structural variants alone. Supplementary table provides a comprehensive review of all the clinical information available on this cohort. Below is extracted information from these tables.

\section{Genotype-phenotype correlation in SIN3A}

Most of the patients in this cohort $(25 / 28 ; 89 \%)$ have truncating or frameshift variants in SIN3A except three patients with missense variants (Patients 4, 18 and 26) which have been classed as a class 4 (likely pathogenic) variant based on a combination of evidence as previously described (see variant table for further information). It is likely that as previously described haploinsufficiency is the predominant likely mechanism in SIN3A-related disorder but it may be that the phenotype may differ depending on the nature of the SIN3A variant. From the evidence gathered so far, there is no apparent correlation between severity of phenotype and genotype.

\section{Neurodevelopment}

As noted in our earlier study [1], the overall intellectual disability seems to be mild, with 11 of the 16 patients (69\%) over the age of eight years having either no ID or mild ID. The low prevalence of severe intellectual disability different from the neurodevelopmental phenotype of moderate or severe intellectual disability associated with 15q24 microdeletion syndrome, suggests that additional genes contribute to the cognitive phenotype in the 15q24 deletion syndrome [15].

The median intelligence is relatively high with IQ of 74 . Interestingly, all patients in whom intelligence was tested reported a higher verbal than performal intelligence score. This is an important finding that clinicians should be aware of, since disharmonic intelligence profiles easily lead to overestimating of the self-management capabilities of patients. A further study is planned to undertake detailed psychometric assessments and IQ measurements in this cohort.

\section{Behavioural phenotype}

Overall, a third of the cohort had a psychiatric or behavioural condition reported, including ADHD, aggressive behaviour, OCD, depression, psychosis, anxiety and schizoaffective disorder. In three patients, ASD was concurrent with a psychiatric diagnosis. This is a significant finding, since such neuropathology has a significant impact on the quality of life of patients especially in those adults with milder neurodevelopmental phenotypes. Knowing that 
patients with variants in the SIN3A gene are at risk for such concerns, early intervention is important to ensure optimal treatment and outcomes.

Of those patients with psychiatric disease, only two had brain imaging done. Interestingly, both those MRIs showed ventriculomegaly, while one also showed delayed myelination. The younger patients in the cohort did not have any neuroimaging done given the milder clinical presentation. However, it is likely that once the diagnosis of a $\operatorname{SIN} 3 A$ related disorder is made, imaging of the brain should be offered in the context of neurological symptoms rather than routine work-up.

\section{Craniofacial dysmorphism}

Our previous study presented evidence for a characteristic facial appearance associated with SIN3A. Some of the patients in this cohort have clear similarities. As with many of the mild and variable neurodevelopmental phenotypes, it remains to be seen whether the facial gestalt is easily identifiable in clinical practice. However, there appears to be a common, emerging facial phenotype with a tall, broad forehead, down-slanting palpebral fissures, triangular face with a pointed chin and a thin upper lip, based on the patient's photographs (both included and unpublished but shared with the authors due to parental consent for publication of photos being declined).

Interestingly, one patient was first suspected of progeria, because of the typical shape of his neurocranium (Patient 25: J). Following genetic testing, he was diagnosed with a variant in SIN3A, demonstrating that this may be part of the spectrum of the syndrome. Patient 12: G also appears to have a progeric face. Sparse hair and reduced subcutaneous tissue was reported in $3 / 28(10 \%)$ of patients in this study and note the progeric appearance in at least two of the patients in this cohort. However, no other ectodermal features were identified. This leads to the possibility of differential diagnoses including progeroid group of conditions; however, SIN3A-related disorder does not appear to present predominantly with a progeroid phenotype from the large cohort described here.

\section{Other clinical manifestations}

$14 / 28(50 \%)$ patients in our study population had either epilepsy, hypotonia, or both. Two out of the three patients with epilepsy who had a brain MRI (of a total of five patients with epilepsy) had abnormalities found. None of the patients with epilepsy from this cohort reported any psychiatric disease.

Interestingly, other commonly reported symptoms in patients with intellectual disability were not reported in our patient population. Constipation for instance, was only reported in one patient, while hearing loss and refraction abnormalities were also not as prevalent as in other intellectual disability cohorts or the $15 \mathrm{q} 24$ microdeletion syndrome [15, 16].

Further information also needs to be collected to ascertain whether malignancy is a significant association or merely an observation with a large cohort of patients with Witteveen-Kolk Syndrome. In addition, as described above, additional features appear to be emerging from the larger cohort of patients published here and further follow-up is required to see if this is a consistent part of the phenotype.

\section{Conclusion}

Patients with disease causing variants in SIN3A usually have mild global developmental delay/ID, with some even having tested IQs in the normal range with variable penetrance. There are similar facial features in around half of patients for which a targeted molecular evaluation would be feasible. However, it is likely that diagnostic evaluation and identification of SIN3A variants in suspected individuals will be performed using large ID panels or WES/WGS. There is evidence to suggest these patients are at risk for psychiatric- and neurological conditions and therefore, a multidisciplinary team approach should be considered in caring for these patients. Data collected so far seems to suggest additional features such as hypotonia, seizures along with the previously well described neurodevelopmental association with this disorder.

There is no apparent genotype-phenotype correlation and/ or missense variant hot spot within SIN3A and the missense variants appear to be distributed throughout the gene based on observation of this cohort. As expected, majority of patients in published literature and this cohort appear to have truncating variants reinforcing haploinsufficiency as likely mechanism of pathogenicity, although SIN3A missense variants affecting critical functional domain in SIN3A also appear to be associated with disease. Further studies of this nature are required to ascertain clinical correlation in this disorder.

Acknowledgements We are grateful to the patients and their families for their cooperation. This study makes use of data generated by the DECIPHER Consortium. A full list of centres who contributed to the generation of the data is available from https://decipher.sanger.ac.uk/ and via email from decipher@sanger.ac.uk. Funding for the project was provided by the Wellcome Trust and by grants from the Netherlands Organization for Health Research and Development (ZonMw grant 91718310 and the Dutch Scientific Organization (NWO, grant NWA 1160.18.320). WKC is supported by grants from SFARI and the JPB Foundation

DDD statement The DDD study presents independent research commissioned by the Health Innovation Challenge Fund [grant number 
HICF-1009-003]. This study makes use of DECIPHER (http:// decipher.sanger.ac.uk), which is funded by the Wellcome Trust. See Nature PMID: 25533962 or www.ddduk.org/access.html for full acknowledgement. We would also like to thank all the families for consenting to this publication.

Author contributions $\mathrm{MB}$ and TK designed and supervised the study; MB was responsible for collection of data from UK cohort of patients whilst TK was responsible for data collection from Dutch and international cohort; SA, AD and RR performed all data collection and molecular interpretation of SIN3A variants; all authors contributed to clinical or molecular data collection and approval of submitted manuscript.

\section{Compliance with ethical standards}

Conflict of interest The authors declare that they have no conflict of interest.

Ethics statement This study was performed in adherence to the principles set out in the Declaration of Helsinki. The DDD study has UK Research Ethics Committee approval (10/H0305/83, granted by the Cambridge South REC, and GEN/284/12 granted by the Republic of Ireland REC). Informed consent for publication of clinical and molecular data and inclusion of photos where applicable was obtained from all patients and/ or their guardians included in this study.

Publisher's note Springer Nature remains neutral with regard to jurisdictional claims in published maps and institutional affiliations.

Open Access This article is licensed under a Creative Commons Attribution 4.0 International License, which permits use, sharing, adaptation, distribution and reproduction in any medium or format, as long as you give appropriate credit to the original author(s) and the source, provide a link to the Creative Commons license, and indicate if changes were made. The images or other third party material in this article are included in the article's Creative Commons license, unless indicated otherwise in a credit line to the material. If material is not included in the article's Creative Commons license and your intended use is not permitted by statutory regulation or exceeds the permitted use, you will need to obtain permission directly from the copyright holder. To view a copy of this license, visit http://creativecommons. org/licenses/by/4.0/.

\section{References}

1. Witteveen JS, Willemsen MH, Dombroski TC, van Bakel NH, Nillesen WM, van Hulten JA, et al. Haploinsufficiency of MeCP2interacting transcriptional co-repressor SIN3A causes mild intellectual disability by affecting the development of cortical integrity. Nat Genet. 2016;48:877-87.

2. Stessman HA, Xiong B, Coe BP, Wang T, Hoekzema K, Fenckova M, et al. Targeted sequencing identifies 91 neurodevelopmental-disorder risk genes with autism and developmental-disability biases. Nat Genet. 2017;49:515-26.

3. Narumi-Kishimoto Y, Araki N, Migita O, Kawai T, Okamura K, Nakabayashi $\mathrm{K}$, et al. Novel SIN3A mutation identified in a Japanese patient with Witteveen-Kolk syndrome. Eur J Med Genet. 2019;62:103547.

4. Mefford HC, Rosenfeld JA, Shur N, Slavotinek AM, Cox VA, Hennekam RC, et al. Further clinical and molecular delineation of the 15 q24 microdeletion syndrome. J Med Genet. 2012;49: $110-8$.

5. Grzenda A, Lomberk G, Zhang JS, Urrutia R. Sin3: master scaffold and transcriptional corepressor. Biochim Biophys Acta. 2009; 1789:443-50.

6. Wright CF, Fitzgerald TW, Jones WD, Clayton S, McRae JF, van Kogelenberg M, et al. Genetic diagnosis of developmental disorders in the DDD study: a scalable analysis of genome-wide research data. Lancet. 2015;385:1305-14.

7. Sobreira N, Schiettecatte F, Valle D, Hamosh A. GeneMatcher: a matching tool for connecting investigators with an interest in the same gene. Hum Mutat. 2015;36:928-30.

8. Firth HV, Richards SM, Bevan AP, Clayton S, Corpas M, Rajan $D$, et al. DECIPHER: database of chromosomal imbalance and phenotype in humans using ensembl resources. Am J Hum Genet. 2009;84:524-33.

9. Richards S, Aziz N, Bale S, Bick D, Das S, Gastier-Foster J, et al. Standards and guidelines for the interpretation of sequence variants: a joint consensus recommendation of the American College of Medical Genetics and Genomics and the Association for Molecular Pathology. Genet Med. 2015;17:405-24.

10. Ellard S, Baple EL, Callaway A, Berry I, Forrester N, Turnbull C, et al. ACGS best practice guidelines for variant classification 2020. Recommendations ratified by ACGS Quality Subcommittee on $04 / 02 / 2020$

11. Sahu SC, Swanson KA, Kang RS, Huang K, Brubaker K, Ratcliff $\mathrm{K}$, et al. Conserved themes in target recognition by the PAH1 and PAH2 domains of the Sin 3 transcriptional corepressor. J Mol Biol. 2008;375:1444-56.

12. He Y, Radhakrishnan I. Solution NMR studies of apo-mSin3A and $\mathrm{mSin} 3 \mathrm{~B}$ reveal that the $\mathrm{PAH} 1$ and $\mathrm{PAH} 2$ domains are structurally independent. Protein Sci. 2008;17:171-5.

13. Pettersen EF, Goddard TD, Huang CC, Couch GS, Greenblatt DM, Meng EC, et al. UCSF Chimera-a visualization system for exploratory research and analysis. J Comput Chem. 2004;25:1605-12.

14. Rydzewska E, Hughes-McCormack LA, Gillberg C, Henderson A, MacIntyre C, Rintoul J, et al. Age at identification, prevalence and general health of children with autism: observational study of a whole country population. BMJ Open. 2019;9:e025904.

15. Mefford H, Shur N, Rosenfeld J, Adam MP, Ardinger HH, Pagon RA et al. 15q24 Microdeletion syndrome. In MP Adam, HH Ardinger, RA Pagon, SE Wallace, LJH Bean, K Stephens \& A Amemiya (Eds.), GeneReviews((R)). Seattle (WA). 1993

16. Chaidez V, Hansen RL, Hertz-Picciotto I. Gastrointestinal problems in children with autism, developmental delays or typical development. J Autism Dev Disord. 2014;44:1117-27.

\section{Affiliations}

Meena Balasubramanian ${ }^{1,2} \cdot$ Alexander J. M. Dingemans $^{3} \cdot$ Shadi Albaba $^{4} \cdot$ Ruth Richardson $^{5} \cdot$ Thabo M. Yates $^{1}{ }^{1} \cdot$ Helen Cox $^{6} \cdot$ Sofia Douzgou ${ }^{7,8} \cdot$ Ruth Armstrong ${ }^{9}$. Francis H. Sansbury $\mathbb{1}^{10} \cdot$ Katherine B. Burke $^{10}$. Andrew E. Fry $\mathbb{D}^{10}$ - Nicola Ragge ${ }^{6,11}$ - Saba Sharif ${ }^{6}$ - Alison Foster ${ }^{6}$ - Annachiara De Sandre-Giovannoli ${ }^{12,13,14}$. Sahar Elouej ${ }^{12} \cdot$ Pradeep Vasudevan $^{15} \cdot$ Sahar Mansour $^{16} \cdot$ Kate Wilson $^{17} \cdot$ Helen Stewart $^{17} \cdot$ Solveig Heide $^{18}$. 
Caroline Nava $^{18} \cdot$ Boris Keren $^{18} \cdot$ Serwet Demirdas $^{19} \cdot$ Alice S. Brooks $^{19} \cdot$ Marie Vincent $\mathbb{D i D}^{20,21} \cdot$ Bertrand Isidor $^{20,21}$. Sebastien Küry $\mathbb{D}^{20,21} \cdot$ Meyke Schouten $^{3} \cdot$ Erika Leenders $^{3} \cdot$ Wendy K. Chung $^{22} \cdot$ Arie van Haeringen $^{23}$. Thomas Scheffner ${ }^{24}$ • Francois-Guillaume Debray ${ }^{25}$. Susan M. White ${ }^{26,27}$. Maria Irene Valenzuela Palafoll (iD ${ }^{28}$. Rolph Pfundt ${ }^{3} \cdot$ Ruth Newbury-Ecob ${ }^{29} \cdot$ Tjitske Kleefstra $^{3}$

1 Sheffield Clinical Genetics Service, Sheffield Children's NHS Foundation Trust, Sheffield, UK

2 Academic Unit of Child Health, Department of Oncology \& Metabolism, University of Sheffield, Sheffield, UK

3 Department of Human Genetics, Donders Institute for Brain, Cognition and Behavior, Radboud University Medical Center, Nijmegen, the Netherlands

4 Sheffield Diagnostic Genetics Service, Sheffield Children's NHS Foundation Trust, Sheffield, UK

5 Northern Genetics Service, Newcastle upon Tyne Hospitals NHS Trust, Newcastle, UK

6 West Midlands Regional Clinical Genetics Service and Birmingham Health Partners, Birmingham Women's and Children's Hospitals NHS Foundation Trust, Birmingham, UK

7 Manchester Centre for Genomic Medicine, Saint Mary's Hospital, Manchester University NHS Foundation Trust, Manchester, UK

8 Division of Evolution and Genomic Sciences, School of Biological Sciences, Faculty of Biology, Medicines and Health, University of Manchester, Manchester, UK

9 East Anglian Medical Genetics Service, Addenbrooke's Hospital, Cambridge, UK

10 All Wales Medical Genomics Service, NHS Wales Cardiff and Vale University Health Board, Institute of Medical Genetics, University Hospital of Wales, Cardiff, UK

11 Department of Biological and Medical Sciences, Oxford Brookes University, Oxford, UK

12 Aix Marseille Univ, INSERM, MMG, U1251 Marseille, France

13 Department of Medical Genetics, La Timone Children's Hospital, Marseille, France

14 Biological Resource Center (CRB-TAC), Assistance Publique Hôpitaux de Marseille, La Timone Children's Hospital, Marseille, France
15 Leicester Clinical Genetics Service, University Hospitals of Leicester NHS Trust, Leicester, UK

16 Clinical Genetics Service, St George's University Hospitals NHS Foundation Trust, London, UK

17 Oxford Centre for Genomic Medicine, Nuffield Orthopaedic Centre, Oxford University Hospitals NHS Foundation Trust, Oxford, UK

18 Clinical Genetics Service, GH Pitié-Salpêtrière, Pitié Salpêtrière Hospital, APHP Sorbonne University, Paris, France

19 Department of Clinical Genetics, Erasmus Medical Centre, Erasmus University, Rotterdam, the Netherlands

20 Service de Génétique Médicale, CHU de Nantes, 44000 Nantes, France

21 Inserm, CNRS, Univ Nantes, l'institut du thorax, 44000 Nantes, France

22 Departments of Pediatrics and Medicine, Columbia University, New York, USA

23 Department of Clinical Genetics, Leiden University Medical Center, Leiden, the Netherlands

24 Klinik für Kinder- und Jugendmedizin, Perinatal- und Stoffwechselzentrum, Reutlingen, Germany

25 Metabolic Unit-Department of Medical Genetics, CHU \& University Liège Domaine L Sart-Tilman Bât B35, B-4000 Liège, Belgium

26 Victorian Clinical Genetics Services, Murdoch Children's Research Institute, Melbourne, VIC, Australia

27 Department of Paediatrics, University of Melbourne, Melbourne, VIC, Australia

28 Department of Clinical and Molecular Genetics, University Hospital Vall d’Hebron and Medicine Genetics Group, Valle Hebron Research Institute, Barcelona, Spain

29 University Hospitals Bristol NHS Foundation Trust, Clinical Genetics, St. Michael's Hospital, Bristol, UK 